\title{
Análisis de las diferencias de rendimiento del alumnado de centros urbanos y no urbanos a partir de los resultados de los premios extraordinarios de educación secundaria obligatoria
}

\author{
Analysis of Performance Differences among Students in Urban and Non-urban Centers \\ Based on Compulsory Secondary Education Special Awards
}

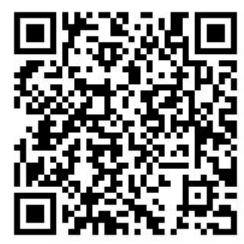

Rocío Chao-Fernández

Universidad de A Coruña

A Coruña, España

rocio.chao@udc.es

Francisco Jesús Ferreiro-Seoane ${ }^{2}$

Universidad de Santiago

Santiago de Compostela, España

franciscojesus.ferreiro@usc.es

María Dorinda Mato-Vázquez 3

Universidad de A Coruña

A Coruña, España

m.matov@udc.es

Recibido 25 de julio de 2014 • Corregido 6 de agosto de 2015 • Aceptado 14 de agosto de 2015

\footnotetext{
${ }^{1}$ Profesora contratada doctora en el Departamento de Composición. Facultad de Ciencias de la Educación. Campus de Elviña, s/n 15071 A Coruña. Además de obtener Premio Extraordinario de Doctorado ha sido galardonada con diferentes premios de innovación educativa a nivel nacional (Mec y Cide, Consellería de Educación, etc.). Una de sus líneas de investigación se centra en analizar los elementos que inciden en el aprendizaje, en busca de una mejora del rendimiento académico, que han generado numerosas publicaciones, entre las que cabe destacar: "Music therapy in adolescent disruptive behaviour", en Procedia - Social and Behavioral Sciences 132, 608-614 (2014). “¿Contribuyen los centros de educación primaria de Galicia a la conservación del folklore?" en Revista Lusófona de Educaçao.

2 Profesor asociado y doctor en el Departamento de Economía Aplicada, Facultad de Dirección y Administración de Empresas de la USC. Sus líneas de investigación se centran actualmente en el campo del emprendimiento y la educación, ha investigado en los campos del desarrollo económico, hacienda pública y economía laboral. Ha sido galardonado tanto por la UNESCO como por la Comisión Europea, por el trabajo realizado en el Plan de Ordenación del Litoral (POL) de Galicia. Ha sido investigador principal de numerosos proyectos y autor de libros y artículos derivados de los campos de investigación citados anteriormente, entre los más recientes: "La rentabilidad económica de los viveros de empresas en Galicia", en Boletín Económico de ICE, 3049, 43-54, 2014; "Los viveros gallegos como instrumento de desarrollo local: Situación actual y líneas futuras de mejora", en Revista Galega de Economía, 20(1), 1-23, 2011 y "The reform of the Local Administration in Spain in Terms of Population", en Journal of US-China Administration, 10(19), 945-957, 2013.

${ }^{3}$ Profesora contratada doctora en Departamento de Pedagogía y Didáctica, Facultad de Ciencias de la Educación, Campus de Elviña, s/n 15071 A Coruña. Sus líneas de investigación se enmarcan en el estudio comparativo de las habilidades procedimentales que se enseñan en ciencias de la naturaleza y en matemáticas en la ESO, que han dado lugar a numerosas publicaciones, como: "Dimensión afectiva hacia la matemática: Resultados de un análisis en educación primaria", en Revista de Investigación Educativa, 32(1), 57-72, 2014. "Influencia de la profesión de los padres en la ansiedad hacia la matemática y su relación con el rendimiento académico en estudiantes de secundaria", en Ciencias Psicológicas 8(1), 69-77, 2014.
} 
doi: http://dx.doi.org/10.15359/ree.19-3.23

URL: http://www.una.ac.cr/educare

CORREO: educare@una.cr

Resumen. Este trabajo analiza cómo inciden, en el rendimiento del alumnado que se presenta al premio extraordinario de educación secundaria obligatoria (ESO) de Galicia, los contextos de enseñanzaaprendizaje referentes a los ámbitos (urbano y no urbano) de las cuatro provincias y las diferentes localidades. La población está formada por 1212 estudiantes una vez que han terminado $4^{\circ}$ de ESO con una calificación media superior a 9 entre los años 2008-2012. A tal objeto, se realizó un análisis comparativo a través de muestras independientes con la prueba de Levene, mediante el paquete estadístico SPSS V. 22.0.0.0. Los resultados indican que, de las 12 calificaciones (lengua gallega, castellana y extranjera, matemáticas y ciencias sociales, geografía e historia) y un examen a elegir entre diversas modalidades (física y química, biología y geología, latín, música, tecnología y educación plástica), en diez de ellas son mejores los procedentes de los centros urbanos $(83,33 \%)$ con diferencias significativas en seis.

Palabras claves. Premio extraordinario, educación secundaria obligatoria, calidad, educación, Galicia, hábitat, urbano y no urbano.

Abstract. This research analyzes how the teaching-learning contexts (urban and non-urban) of four provinces and their different towns influence the school performance of Galician students who apply for the compulsory secondary education (ESO, in Spanish) special award. The sample is of 1,212 students who have finished the 4th level of ESO with an average score greater than or equal to 9 between 2008 and 2012. For such purpose, we made a comparative analysis using independent samples with Levene's test through the SPSS V. 22.0.0.0 statistical package. The results indicate that, out of the 12 scores (Galician, Spanish, foreign language, Mathematics and Social Sciences, Geography and History) and a test to choose from different options (Physics and Chemistry, Biology and Geology, Latin, Music, Technology and Arts), students from urban schools get better results in 10 of them (83.33\%), with significant differences in 6 subjects.

Keywords. Special award, compulsory secondary education, quality, education, Galicia, habitat, urban, non-urban.

Cualquier sistema educativo de calidad persigue una buena formación de su alumnado. Para ello, es necesario que este obtenga los mejores resultados posibles, por lo que, en los últimos años, la mayoría de los países han desarrollado sistemas para la evaluación del aprendizaje de sus discentes en los diferentes niveles.

A este respecto, distintas investigaciones como las realizadas por Broc (2006), Calero y Escardíbul (2007), García, Rubio y Bouachra (2008), Lobato y Alonso (2002), Pascual (2006) y Sánchez (2008), aportan diversas justificaciones a las diferencias de rendimiento entre adolescentes que apuntan a las circunstancias personales, a su origen, a las familias, a las características socioeconómicas, o a la motivación del alumnado hacia la escuela, entre otras causas.

Por su parte, Kleinfeld, McDiarmid y Hagstrom (1985) y Wößmann (2010) sostienen que el hábitat donde se encuentran los discentes es determinante a la hora de explicar las calificaciones, es decir, que el ámbito influye en el rendimiento estudiantil de la educación 
secundaria obligatoria (ESO). El concepto "hábitat" se refiere al emplazamiento geográfico relativo a la vida del alumnado: espacio urbano o ciudad, y espacio no urbano o rural.

En el presente trabajo queremos averiguar hasta qué punto los estudios realizados por los autores anteriormente citados coinciden con los nuestros y en qué disciplinas. Consideramos que, de haber diferencias, puede ser de utilidad para el profesorado y profesionales de la educación a la hora de afrontar las programaciones y el proceso de enseñanza aprendizaje del alumnado.

\section{El premio extraordinario de la ESO en Galicia}

La Consellería de Educación en la Comunidad Autónoma de Galicia convoca todos los años, desde el curso 2007-2008, los premios extraordinarios de educación secundaria obligatoria (Xunta de Galicia, 2012a, b).

Cada año se pueden conceder hasta un máximo de 20 premios al alumnado que haya obtenido las mejores calificaciones en las diferentes pruebas y cada premio estará dotado con $750 €$ y un diploma acreditativo. Pueden optar a participar quienes hayan obtenido una media igual o superior a 9,00 puntos.

La prueba consta de dos partes: la primera trata diversas cuestiones referidas a lengua gallega y literatura, lengua castellana y literatura y primera lengua extranjera. En la segunda parte, el alumnado dará respuesta a diversas cuestiones de tres materias del currículo de cuarto curso: ciencias sociales, geografía e historia, matemáticas y una a escoger de entre biología y geología, educación plástica y visual, física y química, latín, música y tecnología.

En este estudio se analizan los resultados de estos premios correspondientes a cuatro cursos escolares, concretamente del 2008 al 2012, con un doble enfoque: por una parte se realiza un análisis de las calificaciones obtenidas por estudiantes que se han presentado a los exámenes y han obtenido los premios y, por otra, se exploran las diferencias en algunas variables seleccionadas como son el ámbito urbano y no urbano a través de las asignaturas obligatorias y optativas a las que tienen que hacer frente en dichas pruebas. El documento finaliza con un resumen de los hallazgos más importantes y se avanza en la explicación de estos.

Los datos del estudio han sido facilitados por la Consellería de Educación de la Xunta de Galicia, previa entrevista concertada con el responsable de este organismo, con competencias en educación en la comunidad autónoma. La información tiene, por tanto, carácter oficial y garantiza la calidad de la información en la que se basan las conclusiones obtenidas. 
doi: http://dx.doi.org/10.15359/ree.19-3.23

URL: http://www.una.ac.cr/educare

CORREO: educare@una.cr

\section{El hábitat del centro y sus efectos sobre el rendimiento}

Todo el alumnado es "diverso":

ante los procesos de aprendizaje que se desarrollan en la institución escolar, porque son diferentes en cuanto a aptitudes, intereses, motivaciones, capacidades, ritmos de maduración y estilos de aprender, experiencias y conocimientos previos, entornos sociales y culturales. Estos aspectos conforman tipologías y perfiles en [los estudiantes] que deben determinar en gran medida la planificación y la acción [educativa]. (Fernández, Jorge y Martín, 2006, p. 40)

Indudablemente, en estas tipologías debemos tener en cuenta, entre otras, la del alumnado de alto rendimiento a la que pertenece la población de nuestro estudio (Mato, Chao y Ferreiro, 2015).

Por otra parte, hemos de reconocer que son pocos los estudios que han abordado el efecto de la localización urbana o no urbana de las escuelas en el rendimiento del alumnado, quizás por dos motivos: en primer lugar, porque la definición de zona urbana y no urbana varía de forma considerable entre los países; en segundo lugar, y desde nuestro punto de vista más importante, porque detrás de estas cifras se oculta la incidencia del nivel socioeconómico y cultural de las familias del estudiantado (Häkkinen, Kirjavainen y Uusitalo, 2003; Morales, Benítez y Agustín, 2013; Wößmann, 2003). Está ampliamente constatado que los factores citados condicionan su progreso educativo y los resultados que obtienen (Casullo, 2002; Lizasoain, Joaristi, Lucas y Santiago, 2006; Serrano, Bojórquez, Vera y Ramos, 2009) evidencian una clara asociación entre el rendimiento académico y el nivel socioeconómico.

Con respecto a la relación rendimiento alumnado-localización urbana o no urbana de las escuelas no parece haber un consenso explícito. Así, Santos (2007) y Wößmann (2010) no encuentran diferencias significativas en las calificaciones de estudiantes de escuelas urbanas y no urbanas. Por el contrario, Chiswick y DebBurman (2004) y Meunier (2011) descubren que la variable localización es significativa a la hora de explicar el rendimiento escolar.

Obviamente, deberíamos considerar la posibilidad de que en esas diferencias tengan mucho que ver las características estudiantiles de las escuelas situadas en el entorno de cada una de las zonas urbanas/no urbanas; así como las tipologías y particularidades de sus familias. De hecho, Young (1998) sostiene que las desigualdades académicas se deben a las especiales y exclusivas peculiaridades de las mencionadas variables (urbana/no urbana). En esta línea, Wößmann (2010), a partir de los datos obtenidos en el test del Progress in International Reading 
Literacy Study en 2001, manifiesta que estudiantes de poblaciones con más de tres mil habitantes superan en más de 26 puntos los resultados obtenidos por los de las zonas rurales.

Por otra parte, el informe PISA, elaborado por la Organización para la Cooperación y el Desarrollo Económicos (OCDE) (2010) con la información recopilada tanto en 2009 como en 2012, analiza la importancia de la localización de la escuela para explicar las diferencias en los resultados obtenidos en las pruebas de lectura. No obstante, tiene en cuenta las características socioeconómicas de las familias de sus estudiantes.

\section{Metodología}

Tomando como referencia lo reseñado anteriormente, el objetivo de este trabajo es analizar si existen diferencias significativas en el rendimiento del alumnado que se ha presentado al premio extraordinario de la ESO en los años 2008-09, 2009-10, 2010-11 y 2011-12 del medio urbano y no urbano de la comunidad autónoma de Galicia.

\section{Población}

La población está formada por 1212 estudiantes, que suponen la totalidad del alumnado que se presentó a esta prueba en el período 2008-2012, una vez que han terminado $4^{\circ}$ curso de educación secundaria obligatoria con una calificación media superior a 9. Consideramos que esos datos correspondientes a cuatro cursos académicos son suficientes para poder estudiar el rendimiento por hábitat.

A la hora de clasificar discentes por hábitat se ha estimado el criterio de habitantes, de tal manera que se han considerado urbanas las siete ciudades más importantes de Galicia (con más de 50.000 habitantes) y el resto no urbanas. Con este criterio objetivo, se supone alumnado urbano el que se ha presentado a la prueba vinculado a un colegio ubicado en las ciudades de Vigo, A Coruña, Ourense, Lugo, Santiago de Compostela, Pontevedra y Ferrol, lo que significa 796 discentes $(59,1 \%)$, frente al alumnado del resto de los 309 municipios gallegos (no urbano o rural) que con 496 estudiantes representa el 40,9\%.

Para analizar los resultados de las pruebas, se han establecido varias hipótesis que atienden las materias de las que se tiene que examinar el estudiantado para acceder a los premios extraordinarios (tabla 1). A tal objeto se realizó un análisis comparativo a través de muestras independientes con la prueba de Levene mediante el paquete estadístico SPSS v.22.0. 
doi: http://dx.doi.org/10.15359/ree.19-3.23

URL: http://www.una.ac.cr/educare

CORREO: educare@una.cr

Tabla 1

Hipótesis formuladas

\begin{tabular}{|c|c|}
\hline Hipótesis & Formulación. \\
\hline Hipótesis 1 & Existen diferencias significativas en las calificaciones medias generales en función del hábitat. \\
\hline Hipótesis 2 & Existen diferencias significativas en la calificación media de lengua extranjera ${ }^{4}$ en función del hábitat. \\
\hline Hipótesis 3 & Existen diferencias significativas en la calificación media de lengua castellana en función del hábitat. \\
\hline Hipótesis 4 & Existen diferencias significativas en la calificación media de lengua gallega en función del hábitat. \\
\hline Hipótesis 5 & $\begin{array}{l}\text { Existen diferencias significativas en la calificación media de ciencias sociales y geografía e } \\
\text { historia }(\mathrm{CSGH}) \text { en función del hábitat. }\end{array}$ \\
\hline Hipótesis 6 & Existen diferencias significativas en la calificación media de matemáticas en función del hábitat. \\
\hline Hipótesis 7 & Existen diferencias significativas en la calificación media de biología y geología en función del hábitat. \\
\hline Hipótesis 8 & Existen diferencias significativas en la calificación media de física y química en función del hábitat. \\
\hline Hipótesis 9 & Existen diferencias significativas en la calificación media de latín en función del hábitat. \\
\hline Hipótesis 10 & Existen diferencias significativas en la calificación media de educación plástica en función del hábitat. \\
\hline Hipótesis 11 & Existen diferencias significativas en la calificación media de música en función del hábitat. \\
\hline Hipótesis 12 & Existen diferencias significativas en la calificación media de tecnología en función del hábitat. \\
\hline
\end{tabular}

${ }^{4}$ Se podría considerar que es de inglés, pero existen estudiantes que se han decantado por el idioma francés.

\section{Análisis de los resultados}

Primera hipótesis: Existen diferencias significativas en las calificaciones medias generales en función del hábitat.

La primera observación, antes de hacer un análisis por hábitat, es que las calificaciones en general no son elevadas, teniendo en cuenta que se trata de estudiantes que han obtenido una media de sobresaliente, estas son de aprobado (tabla 2), lo que supone la dificultad de la pruebas realizadas. Las calificaciones en Galicia oscilan desde 0 hasta 4 suspenso, 5 aprobado, 6 bien, 7-8 notable, 9 y 10 sobresaliente.

Si nos centramos en las diferencias entre los dos grupos analizados, podemos atisbar una clara corriente: los ubicados en zonas urbanas adquieren mejores calificaciones de media que los que viven en zonas no urbanas en la mayoría de las asignaturas y años analizados. 
Tabla 2

Calificaciones medias de los exámenes clasificados por hábitat del centro (2008-12)

\begin{tabular}{lccccccc}
\hline & L. Extranjera & L. Castellana & L. Gallega & CSGH & Matemáticas & Optativa & General \\
\hline 2008/09 & $\mathbf{7 , 7 2}$ & $\mathbf{5 , 9 1}$ & $\mathbf{6 , 5 5}$ & $\mathbf{4 , 3 5}$ & $\mathbf{5 , 4 8}$ & $\mathbf{4 , 5 9}$ & $\mathbf{5 , 7 7}$ \\
Urbana & $\mathbf{7 , 9 4}$ & 6,00 & 6,64 & 4,50 & 5,90 & 4,52 & 5,92 \\
No urbana & 7,42 & 5,78 & 6,44 & 4,15 & 4,91 & 4,70 & 5,57 \\
2009/10 & $\mathbf{7 , 8 2}$ & $\mathbf{4 , 6 3}$ & $\mathbf{5 , 6 4}$ & $\mathbf{5 , 4 3}$ & $\mathbf{3 , 5 8}$ & $\mathbf{4 , 8 7}$ & $\mathbf{5 , 3 3}$ \\
Urbana & $\mathbf{7 , 9 6}$ & 4,64 & 5,73 & 5,64 & 3,86 & 5,21 & 5,51 \\
No urbana & $\mathbf{7 , 6 3}$ & 4,62 & 5,51 & 5,14 & 3,20 & 4,39 & 5,08 \\
2010/11 & $\mathbf{7 , 4 2}$ & $\mathbf{5 , 5 0}$ & $\mathbf{4 , 9 4}$ & $\mathbf{5 , 2 4}$ & $\mathbf{3 , 2 0}$ & $\mathbf{4 , 4 9}$ & $\mathbf{5 , 1 3}$ \\
Urbana & $\mathbf{7 , 6 3}$ & 5,74 & 5,13 & 5,50 & 3,45 & 4,81 & 5,38 \\
No urbana & $\mathbf{6 , 9 6}$ & 4,95 & 4,53 & 4,68 & 2,66 & 3,78 & 4,59 \\
2011/12 & $\mathbf{7 , 8 5}$ & $\mathbf{6 , 0 6}$ & $\mathbf{5 , 6 9}$ & $\mathbf{3 , 6 8}$ & $\mathbf{4 , 7 2}$ & $\mathbf{3 , 6 7}$ & $\mathbf{5 , 2 7}$ \\
Urbana & $\mathbf{6 , 0 8}$ & $\mathbf{6 , 2 7}$ & 5,65 & 3,92 & 4,94 & 3,62 & 5,40 \\
No urbana & $\mathbf{7 , 5 6}$ & 5,78 & 5,73 & 3,37 & 4,43 & 3,73 & 5,10 \\
\hline Total general & $\mathbf{7 , 7 3}$ & $\mathbf{5 , 5 1}$ & $\mathbf{5 , 7 8}$ & $\mathbf{4 , 6 3}$ & $\mathbf{4 , 3 5}$ & $\mathbf{4 , 4 1}$ & $\mathbf{5 , 4 0}$ \\
\hline Urbana & $\mathbf{7 , 9 2}$ & 5,64 & 5,83 & 4,87 & 4,60 & 4,54 & 5,56 \\
No urbana & $\mathbf{7 , 4 6}$ & 5,33 & 5,71 & 4,28 & 3,98 & 4,21 & 5,16 \\
\hline
\end{tabular}

En la tabla 3 se pueden ver, más específicamente, las diferencias de calificaciones medias entre los pertenecientes a los centros urbanos y no urbanos. En efecto, existe una diferencia positiva a favor de los primeros: el promedio de calificación para el período considerado (2008-2012) es superior en 0,40 puntos a su favor y en todas las asignaturas, aunque no en todos los años.

Tabla 3

Diferencias de calificaciones medias entre no urbano y urbano para el período 2008-12

\begin{tabular}{lccccccc}
\hline Diferencias & L. Extranjera & L. Castellana & L. Gallega & CSGH & Matemáticas & Optativa & General \\
\hline $\mathbf{2 0 0 8 / 0 9}$ & 0,52 & 0,22 & 0,20 & 0,36 & 0,98 & $-0,19$ & 0,35 \\
$\mathbf{2 0 0 9 / 1 0}$ & 0,33 & 0,02 & 0,22 & 0,50 & 0,66 & 0,83 & 0,43 \\
$\mathbf{2 0 1 0 / 1 1}$ & 0,67 & 0,79 & 0,60 & 0,82 & 0,79 & 1,03 & 0,78 \\
$\mathbf{2 0 1 1 / 1 2}$ & 0,51 & 0,49 & $-0,08$ & 0,54 & 0,50 & $-0,11$ & 0,30 \\
\hline Total general & $\mathbf{0 , 4 6}$ & $\mathbf{0 , 3 1}$ & $\mathbf{0 , 1 2}$ & $\mathbf{0 , 5 8}$ & $\mathbf{0 , 6 2}$ & $\mathbf{0 , 3 3}$ & $\mathbf{0 , 4 0}$ \\
\hline
\end{tabular}


doi: http://dx.doi.org/10.15359/ree.19-3.23

URL: http://www.una.ac.cr/educare

CORREO: educare@una.cr

Estos resultados parecen concordar con Chiswick y DebBurman (2004) y Meunier (2011), quienes encontraron significativa la variable de localización urbana/no urbana a la hora de explicar el rendimiento estudiantil.

Para verificar el cumplimento de la primera hipótesis realizamos el test de Levene (anexos A y B) y comprobamos que el $p$-valor es de casi cero, lo que nos lleva a afirmar que existen diferencias significativas en las calificaciones medias generales en función del hábitat.

Segunda hipótesis: Existen diferencias significativas en la calificación media de lengua extranjera en función del hábitat.

En la tabla 2, se puede ver que lengua extranjera es la asignatura que alcanza la mayor calificación media, así como una de las que menor variabilidad anual presenta. Podemos, por tanto, afirmar que es después de matemáticas y CSGH una de las materias donde la diferencia resulta ser más elevada (tabla 3 ).

Procedemos como en el caso de la hipótesis anterior $y$, a través del test de Levene, se evidencia su cumplimiento (anexos A y B). Ciertamente coinciden estos datos con los mencionados a nivel teórico al hallar diferencias entre las calificaciones del alumnado de diferentes hábitats.

A continuación analizaremos las dos lenguas oficiales que se estudian en Galicia. El informe PISA 2009 analiza parcialmente la importancia de la localización en estas materias y sitúa las diferencias entre la puntuación obtenida en las zonas menos pobladas y las más pobladas alrededor del $4 \%$ (OCDE, 2010).

Tercera hipótesis: Existen diferencias significativas en la calificación media de lengua castellana en función del hábitat.

Con respecto a esta hipótesis debemos señalar que existen diferencias entre discentes de distinta procedencia, salvo los datos del año 2009/2010, donde se mantienen muy similares (tabla 3).

En el anexo B, se puede observar el cumplimiento de esta hipótesis con un resultado altamente significativo, con lo cual, el alumnado denominado urbano, tiene una mayor calificación en lengua castellana, lo que coincide con las conclusiones del informe PISA.

Cuarta hipótesis: Existen diferencias significativas en la calificación media de lengua gallega en función del hábitat.

En cuanto a esta, hay que destacar que a pesar de tener una variabilidad interanual similar a lengua castellana, los resultados obtenidos por los grupos analizados son mucho más similares, con una diferencia neta de los años estudiados de 0,12. 
En el anexo B se observa el incumplimiento de la hipótesis, con un estadístico p-valor alto. En este caso no se cumplen las conclusiones del informe PISA. Este incumplimiento invalida la creencia de que, en Galicia, el alumnado no urbano domina la lengua gallega en mayor nivel que el urbano.

Quinta hipótesis: Existen diferencias significativas en la calificación media de ciencias sociales y geografía e historia (CSGH) en función del hábitat.

En la tabla 2 se puede percibir que es una de las materias con una media más baja, $(4,63)$, junto con matemáticas. También es revelador que sea una de las asignaturas donde las diferencias entre el alumnado de las distintas zonas es más destacable $(0,58)$. Estas observaciones concuerdan con las obtenidas por Young (1998), quien sostiene que las diferencias entre el rendimiento de estudiantes de escuelas urbanas y no urbanas se deba a sus características, a sus familias y a las propias escuelas.

Con los datos observados (anexos $A$ y B) queda reflejado el cumplimiento de la hipótesis a favor del alumnado urbano con un resultado altamente significativo Sig (bila)=0,000.

Sexta hipótesis: Existen diferencias significativas en la calificación media de matemáticas en función del hábitat.

Esta materia es la que presenta las peores calificaciones medias registradas en la prueba extraordinaria. También se aprecia una alta variabilidad interanual. Además, es la asignatura donde las diferencias en el estudiantado urbano frente a no urbano se agravan en mayor medida, obteniendo mejores resultados el primero con una diferencia de 0,62 puntos de media y en ningún año menos de 0,5 puntos. Por lo cual, nos hace considerar que también se mostrará relevante dicha diferencia, corroborando el estudio realizado por Wößmann (2003), que encuentra que estudiantes de poblaciones con más de tres mil habitantes superan en más de 26 puntos a los estudiantes de zonas no urbanas.

Al analizar los datos del anexo B concluimos que las varianzas son distintas. Si comprobamos el p-valor, se comprueba que existen diferencias entre estudiantes a favor del urbano.

Hasta el momento hemos examinado los datos de las asignaturas obligatorias. Pasamos a continuación a analizar las materias optativas. La prueba consta de 6 disciplinas con un claro predominio de estudiantes que han elegido biología/geología y física/química, lo que representan conjuntamente el $83,8 \%$ del total de estudiantes.

En la figura 1 observamos la tendencia de elección de optativa entre las muestras analizadas, donde el alumnado urbano opta más por física y química, frente al no urbano que se decanta, principalmente, por biología y geología como primera opción. 
doi: http://dx.doi.org/10.15359/ree.19-3.23

URL: http://www.una.ac.cr/educare

CORREO: educare@una.cr

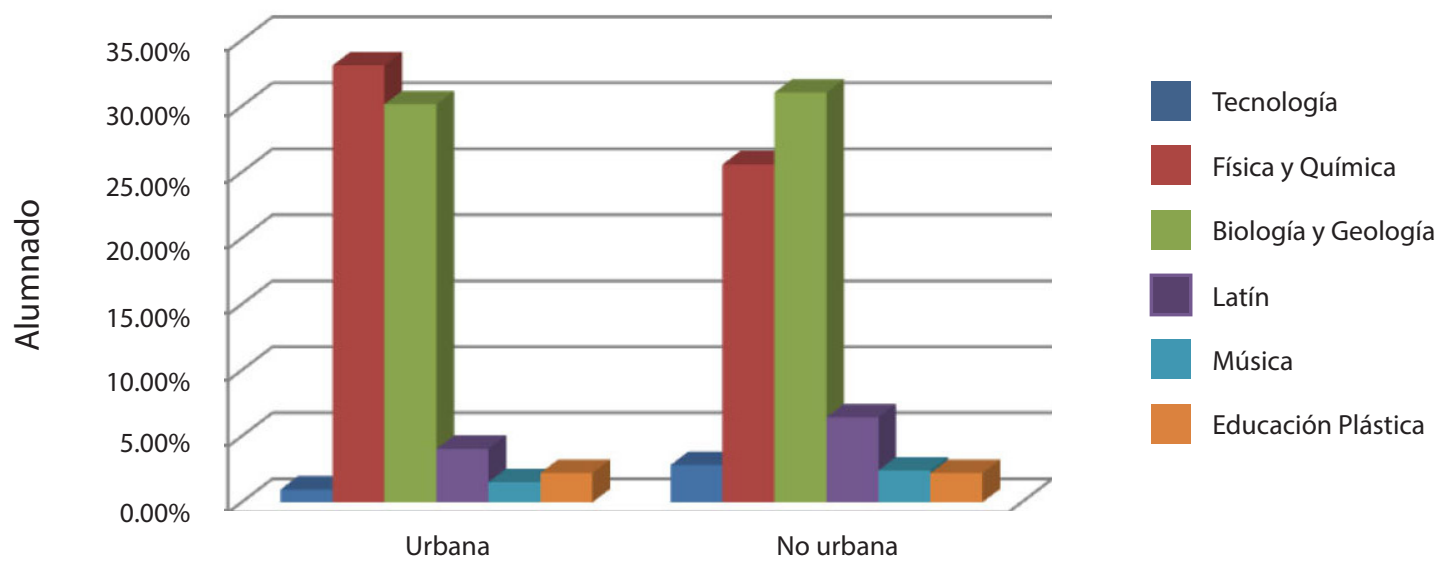

Hábitat

Figura 1. Elección de optativas respecto al total en \% de presentados 2008-12.

A continuación se muestran los resultados alcanzados por estudiantes que se presentaron al examen extraordinario en las materias optativas (tabla 4).

Tabla 4

Calificaciones medias de las optativas clasificadas por hábitat (2008-2012). No aparecen los datos de las optativas del período 2009/2010 porque la Consellería de Educación no los ha facilitado.

\begin{tabular}{|c|c|c|c|c|c|c|}
\hline & Física y Química & Biología y Geología & Latín & Música & Educación Plástica & Tecnología \\
\hline $2008 / 09$ & 3,84 & 4,88 & 6,90 & 5,79 & 4,27 & 4,88 \\
\hline Urbana & 3,75 & 5,03 & 6,67 & 6,42 & 3,50 & 5,50 \\
\hline No urbana & 4,00 & 4,68 & 7,04 & 5,17 & 5,63 & 4,67 \\
\hline $2010 / 11$ & 4,71 & 4,00 & 6,16 & 2,70 & 5,95 & 4,87 \\
\hline Urbana & 5,18 & 4,17 & 6,17 & 2,93 & 5,00 & 6,40 \\
\hline No urbana & 3,38 & 3,68 & 6,12 & 2,53 & 6,27 & 1,80 \\
\hline $2011 / 12$ & 2,67 & 4,00 & 5,98 & 5,38 & 6,67 & 4,09 \\
\hline Urbana & 2,77 & 3,92 & 6,27 & 5,75 & 6,69 & 4,70 \\
\hline No urbana & 2,50 & 4,09 & 5,82 & 5,00 & 6,63 & 3,84 \\
\hline Total general & 3,62 & 4,34 & 6,41 & 4,78 & 5,59 & 4,35 \\
\hline Urbana & 3,79 & 4,41 & 6,34 & 5,35 & 5,19 & 5,30 \\
\hline No urbana & 3,30 & 4,24 & 6,48 & 4,26 & 6,16 & 3,87 \\
\hline
\end{tabular}


Comprobamos que las calificaciones medias de las materias optativas continúan con la misma corriente que en el caso de las obligatorias. En su mayoría consiguen mejores resultados estudiantes de zona urbana; y solo en las materias de latín y educación plástica manifiestan una preferencia discentes de las zonas no urbanas.

La séptima hipótesis: Existen diferencias significativas en la calificación media de biología y geología en función del hábitat.

Analizamos los resultados y se puede ver en los anexos A y B que no existen diferencias significativas entre estudiantes de los distintos hábitats.

Octava hipótesis: Existen diferencias significativas en la calificación media de física y química en función del hábitat.

En la tabla 5 se observan que son las calificaciones más bajas incluso menores que matemáticas, donde en ningún año la media llegó a ser un aprobado. Con los anexos A y $B$ se determina que se cumple la hipótesis; por lo que, con una Sig. (bilateral) $=0.05$, existen diferencias significativas a favor del estudiantado de las grandes ciudades.

Las cuatro hipótesis restantes no se cumplen, ya que las diferencias encontradas en las asignaturas de música, latín, educación plástica y tecnología no son significativas (anexos A y B), a pesar de que existen valores diferenciados según el hábitat (tablas 5 y 6).

Tabla 5

Resultados de las hipótesis formuladas

\begin{tabular}{|c|c|c|c|}
\hline Hipótesis & Formulación & Se cumple & A favor del hábitat \\
\hline H.1 & $\begin{array}{l}\text { Existen diferencias significativas en las calificaciones medias ge- } \\
\text { nerales en función del hábitat. }\end{array}$ & Sí & Urbano \\
\hline H. 2 & $\begin{array}{l}\text { Existen diferencias significativas en la calificación media de len- } \\
\text { gua extranjera en función del hábitat. }\end{array}$ & Sí & Urbano \\
\hline H.3 & $\begin{array}{l}\text { Existen diferencias significativas en la calificación media de len- } \\
\text { gua castellana en función del hábitat. }\end{array}$ & Sí & Urbano \\
\hline H.4 & $\begin{array}{l}\text { Existen diferencias significativas en la calificación media de len- } \\
\text { gua gallega en función del hábitat. }\end{array}$ & No & - \\
\hline H.5 & $\begin{array}{l}\text { Existen diferencias significativas en la calificación media de cien- } \\
\text { cias sociales y geografía e historia ( } \mathrm{CSGH} \text { ) en función del hábitat. }\end{array}$ & Sí & Urbano \\
\hline H. 6 & $\begin{array}{l}\text { Existen diferencias significativas en la calificación media de mate- } \\
\text { máticas en función del hábitat. }\end{array}$ & Sí & Urbano \\
\hline
\end{tabular}

Continúa 
doi: http://dx.doi.org/10.15359/ree.19-3.23

URL: http://www.una.ac.cr/educare

CORREO: educare@una.cr

\begin{tabular}{llcc}
\hline Hipótesis & \multicolumn{1}{c}{ Formulación } & Se cumple & A favor del hábitat \\
\hline H.7 & $\begin{array}{l}\text { Existen diferencias significativas en la calificación media de biolo- } \\
\text { gía y geología en función del hábitat. }\end{array}$ & No & - \\
H.8 & $\begin{array}{l}\text { Existen diferencias significativas en la calificación media de física } \\
\text { y química en función del hábitat. }\end{array}$ & Sí & Urbano \\
H.9 & $\begin{array}{l}\text { Existen diferencias significativas en la calificación media de latín } \\
\text { en función del hábitat. }\end{array}$ & No & - \\
H.10 & $\begin{array}{l}\text { Existen diferencias significativas en la calificación media de edu- } \\
\text { cación plástica y visual en función del hábitat. }\end{array}$ & No & - \\
H.11 & $\begin{array}{l}\text { Existen diferencias significativas en la calificación media de músi- } \\
\text { ca en función del hábitat. }\end{array}$ & No & - \\
H.12 & $\begin{array}{l}\text { Existen diferencias significativas en la calificación media de tec- } \\
\text { nología en función del hábitat. }\end{array}$ & No & - \\
\hline
\end{tabular}

Tabla 6

Resumen del rendimiento del premio extraordinario de la ESO por hábitat y materia (2008-2012)

\begin{tabular}{|c|c|c|}
\hline $\mathrm{N}^{\circ}$ & Rendimiento académico & Materia \\
\hline 1 & $\begin{array}{l}\text { Las zonas urbanas tienen una media superior" signi- } \\
\text { ficativa" en los siguientes exámenes }\end{array}$ & $\begin{array}{l}\text { 1. Calificación global media } \\
\text { 2. Lengua extranjera } \\
\text { 3. Lengua castellana } \\
\text { 4. Ciencias sociales, geografía e historia } \\
\text { 5. Matemáticas } \\
\text { 6. Física y química }\end{array}$ \\
\hline 2 & $\begin{array}{l}\text { Los hábitats no tiene relevancia significativa en los } \\
\text { siguientes exámenes }\end{array}$ & $\begin{array}{l}\text { 1. Lengua gallega } \\
\text { 2. Biología y geología } \\
\text { 3. Latín } \\
\text { 4. Educación plástica } \\
\text { 5. Música } \\
\text { 6. Tecnología }\end{array}$ \\
\hline $2 . a$ & $\begin{array}{l}\text { Las zonas urbanas tienen una media superior "no } \\
\text { significativa" en los siguientes exámenes }\end{array}$ & $\begin{array}{l}\text { 1. Lengua gallega } \\
\text { 2. Biología y geología } \\
\text { 3. Música } \\
\text { 4. Tecnología }\end{array}$ \\
\hline 2.b & $\begin{array}{l}\text { Los hábitats no urbanos tienen una media superior } \\
\text { "no significativa" en los siguientes exámenes }\end{array}$ & $\begin{array}{l}\text { 1. Latín } \\
\text { 2. Educación plástica }\end{array}$ \\
\hline
\end{tabular}




\section{Conclusiones}

A raíz de los resultados se concluye que, teniendo en cuenta el objetivo general, existen diferencias significativas en el rendimiento de estudiantes que se presentan al premio extraordinario de educación secundaria obligatoria (ESO) procedentes del medio urbano y no urbano de la comunidad gallega; lo cual coincide con los estudios realizados, entre otros, por Chiswick y DebBurman (2004); Meunier (2011) y Wößmann (2010).

Cabe mencionar que en diez de las 12 materias consiguen mejores resultados los de la zona urbana (83,33\%). A pesar de que, al examinar la significatividad, se observa en seis de ellas diferencias significativas a favor de los discentes urbanos; en las otras seis, no podemos concluir que un colectivo obtenga mejor rendimiento que el otro.

Se debe hacer constar que en general todas las calificaciones son bajas, a pesar de tratarse de una población con un rendimiento académico excepcional. A este respecto, los peores resultados se revelan en matemáticas y $\mathrm{CSGH}$, manifestándose además en matemáticas la mayor diferencia de resultados entre los dos hábitats, a favor de los centros urbanos.

No forma parte de este estudio analizar las causas de por qué se dan estas diferencias. No obstante, en lo referente a lengua extranjera (hip. 2), la superioridad del alumnado urbano podría justificarse por la existencia de centros privados-concertados en este hábitat. Dato confirmado por los resultados obtenidos en las investigaciones realizadas por Sánchez (2008) y Mato, Chao y Ferreiro (2015), que corroboran que el estudiantado de los centros privadosconcertados obtiene mejor rendimiento en lengua extranjera, ya que establecen como estrategia diferenciadora dar una alta formación en idiomas.

En lo referente a las hipótesis 3, 5 y 6 relativas a lengua castellana, CSGH y matemáticas, también se ve favorecido el estudiantado urbano, mientras que la hipótesis 4, concerniente a lengua gallega, es la única materia obligatoria en la que no se aprecian diferencias a favor de ninguno de ellos.

Se hace necesario precisar que en la comunidad gallega coexisten dos lenguas oficiales (española y gallega). La primera se usa mayoritariamente en el medio urbano y la segunda en el entorno no urbano. Por lo tanto, llama la atención que este dato no se vea reflejado en el estudiantado del medio rural, como así sucede en los del medio urbano.

En cuanto a CSGH sucede algo similar a lengua extranjera, ya que conforme con los estudios citados anteriormente, los resultados en esta disciplina son mejores en el alumnado perteneciente a centros privados-concertados, debido al nivel socio-económico y cultural de las familias. Los contextos rurales cuentan con menos mecanismos de acceso a la cultura (Serrano et al., 2009; Lizasoain et al., 2006).

Con respecto a matemáticas, los estudios realizados por Broc (2006); Calero y Escardíbul (2007) se refieren a la implicación de las familias y la motivación como causas del rendimiento 
doi: http://dx.doi.org/10.15359/ree.19-3.23

URL: http://www.una.ac.cr/educare

CORREO: educare@una.cr

entre otros factores $y$, aunque no hay resultados sobre las diferencias que tengan en cuenta el hábitat, Mato (2014) relaciona los estudios y la profesión de los padres con el rendimiento en esta asignatura. Aunque, este dato debe ser analizado con cautela, ya que nuestra población difiere de manera particular de la estándar.

En lo que atañe a las asignaturas optativas, sólo la hipótesis 8, relativa a la asignatura de Física y Química, es significativamente favorable al alumnado urbano. También es cierto que en las materias optativas la muestra es menor, no resultando ser, por tanto, concluyentes los resultados.

Asimismo, queremos dejar constancia de que no existen evidencias de investigaciones que afirmen o refuten diferencias significativas en relación con el ámbito educativo, ni a nivel nacional ni internacional, respecto al alumnado objeto de nuestro estudio. Por lo mismo, los resultados no son aplicables a todos los estudiantes que finalizan la ESO, dada la peculiaridad de esta población, ni se pueden extrapolar al resto del alumnado de centros de la zona urbana o no urbana.

Queremos finalizar con la siguiente reflexión: la Consellería de Educación en la Comunidad Autónoma de Galicia (Xunta de Galicia, 2012a, b) establece una educación equitativa para todo el alumnado, independientemente de sus condiciones y circunstancias. No obstante, a la luz de los datos obtenidos en nuestra investigación en la que se manifiesta que el alumnado procedente del ámbito urbano que se presenta al Premio Extraordinario de la ESO obtuvo de manera significativa un mejor rendimiento general que el de los centros situados en zona no urbana, no parece que se estén alcanzando los objetivos de ecuanimidad previstos en la Ley.

Indudablemente el abordaje de estas líneas de trabajo genera un potencial formidable en torno a la realidad escolar de nuestra comunidad; se requiere continuar con este tipo de investigaciones para conocer mejor la situación educativa del alumnado gallego y analizar con detenimiento las posibles causas de tales diferencias. Desde aquí nuestro compromiso para buscar las óptimas formas de dar un impulso a este tipo de estudios, a la vez que esperamos que estos resultados puedan ayudar a los grupos profesionales a mejorar y abordar de modo diferente su acción docente.

\section{Referencias}

Broc, M. Á. (Mayo-Agosto, 2006). Motivación y rendimiento académico en alumnos de educación secundaria obligatoria y bachillerato LOGSE. Revista de Educación 340, 379-414. Recuperado de http://www.revistaeducacion.mec.es/re340/re340 14.pdf

Calero, J. y Escardíbul, J.-O. (2007). Evaluación de servicios educativos: El rendimiento en los centros públicos y privados medido en PISA-2003. Hacienda Pública Española, 183(4), 3366. Recuperado de http://www.ief.es/documentos/recursos/publicaciones/revistas/hac pub/183 Evaluacion.pdf

Casullo, M. (Comp.). (2002). Evaluación del bienestar psicológico en lberoamérica. Buenos Aires: Paidós. 
Chiswick, B. R. y DebBurman, N. (2004). Educational Attainment: Analysis by Immigrant Generation [El logro Educativo: Análisis de la Generación Inmigrante]. Economics of Education Review, 23(4), 361-379. doi: http://dx.doi.org/10.1016/j.econedurev.2003.09.002

Fernández, M. C., Jorge, M. y Martín, A. I. (2006). Medidas de atención a la diversidad para alumnado de altas capacidades. TOLEITOLA. Revista de educación del CeP de Toledo, 8, (35-118). Recuperado de http://redined.mecd.gob.es/xmlui/bitstream/ handle/11162/93635/00620093000017.pdf?sequence $=1$

García, F. J., Rubio, M. y Bouachra, O. (Enero-Abril, 2008). Población inmigrante y escuela en España: Un balance de la investigación. Revista de Educación, 345, 23-60. Recuperado de http://www.revistaeducacion.mec.es/re345/re345 02.pdf

Häkkinen, I., Kirjavainen, T. y Uusitalo, R. (2003). School Resources and Student Achievement Revisited: New Evidence from Panel Data [Revisión de los recursos de la escuela y los logros de los estudiantes: nueva evidencia a partir de Panel Data]. Economics of Education Review, 22(3), 329-335. doi: http://dx.doi.org/10.1016/S0272-7757(02)00060-2

Kleinfeld, J. S., McDiarmid, G. W. y Hagstrom, D. (Diciembre, 1985). Alaska's Small Rural High Schools: Are they Working? [Pequeñas escuelas secundarias de Alaska: ¿Están trabajando?] (Serie Iser Report Series No. 58). Alaska: University of Alaska.

Lizasoain, L., Joaristi, L. M., Lucas, J. F. y Santiago, K. (Septiembre de 2006). El efecto contextual del nivel socioeconómico sobre el rendimiento académico en la educación secundaria obligatoria en la Comunidad Autónoma Vasca. X Congreso Nacional de Educación Comparada "El derecho a la educación e un mundo globalizado. Universidad del País Vasco. San Sebastián, España.

Lobato, H. y Alonso, P. (2002). Elementos que influyen en el fracaso escolar. Universidad de Huelva, España.

Mato, M. D. (2014). La afectividad hacia las matemáticas. Madrid: CreateSpace.

Mato, M. D., Chao, R. y Ferreiro, F. J. (2015). Análisis estadístico de los resultados de las pruebas de rendimiento académico delalumnado de laESO, participante en los premios extraordinarios. REOP, 26(1) 25-43. doi: http://dx.doi.org/10.5944/reop.vol.26.num.1.2015.14340

Meunier, M. (Febrero, 2011). Immigration and Student Achievement: Evidence from Switzerland [Inmigración y rendimiento de los estudiantes: El caso de Suiza]. Economics of Education Review, 30(1), 16-38. doi: http://dx.doi.org/10.1016/j.econedurev.2010.06.017

Morales, M., Benítez, M. y Agustín, D. (2013). Habilidades para la vida (cognitivas y sociales) en adolescentes de una zona rural. Revista Electrónica de Investigación Educativa, 15(3), 98113. Recuperado de http://www.scielo.org.mx/pdf/redie/v15n3/v15n3a7.pdf 
doi: http://dx.doi.org/10.15359/ree.19-3.23

URL: http://www.una.ac.cr/educare

CORREO: educare@una.cr

Organización para la Cooperación y el Desarrollo Económicos (OCDE). (2010). PISA 2009 Results: Overcoming Social Background. Equity in Learning Opportunities and Outcomes (Volume II) [Pisa, 2009: La superación del fondo social. La igualdad de oportunidades en el aprendizaje (Volumen II)]. Paris: Autor.

Pascual, B. (2006). Calidad, equidad e indicadores en el sistema educativo español. Pulso, 29, 43-58. Recuperado de http://dspace.uah.es/dspace/handle/10017/5147

Sánchez, A. (2008). Efectos de la inmigración en el sistema educativo: El caso español (Tesis doctoral). Universidad de Barcelona. Barcelona, España.

Santos, M. E. (2007). Calidad de la educación en Argentina: Determinantes y distribución utilizando los resultados de PISA 2000. Bienestar y política social, 3(1), 79-109. Recuperado de http://bienestar.ciss.org.mx/pdf/es/2007/vol 3 num 1407 es.pdf

Serrano, D. M., Bojórquez, C. I., Vera, J. Á. y Ramos, D. Y. (2009). Locus de control y logro académico en dos tipos de ambiente de enseñanza para estudiantes universitarios. Pesquisas e Práticas Psicossociais, 3(2), 167-174. Recuperado de http://www.ufsj.edu.br/portal-repositorio/File/ Encinas etal.pdf

Wößmann, L. (2003). Schooling Resources, Educational Institutions and Student Performance: The International Evidence [Recursos, escolaridad, instituciones educativas y rendimiento de los alumnos: La evidencia internacional]. Oxford Bulletin of Economics and Statistics, 65(2), 117-170. doi: http://dx.doi.org/10.1111/1468-0084.00045

Wößmann, L. (2010). Families, Schools and Primary-School Learning: Evidence for Argentina and Colombia in an International Perspective [Familias y escuelas de Primaria: El caso de Argentina y Colombia desde una perspectiva internacional]. Applied Economics, 42(21), 2645-2665. doi: http://dx.doi.org/10.1080/00036840801964617

Xunta de Galicia. Consellería de Cultura, Educación e Ordenación Universitaria. (2012a). Información del Premio Extraordinario de la ESO 2012. Recuperado de https://www.edu. xunta.es/premioseso

Xunta de Galicia.Consellería de Cultura, Educación eOrdenación Universitaria.(2012b).Avaliación de Diagnóstico. Galicia 2011-2012 (Informe de resultados). Santiago de Compostela.

Young, D. J. (1998). Rural and Urban Differences in Student Achievement in Science and Mathematics: A Multilevel Analysis [Las diferencias rurales y urbanas de Rendimiento Estudiantil en Ciencias y Matemáticas: Análisis multinivel]. School Effectiveness and School Improvement, 9(4), 386-418. doi: 10.1080/0924 


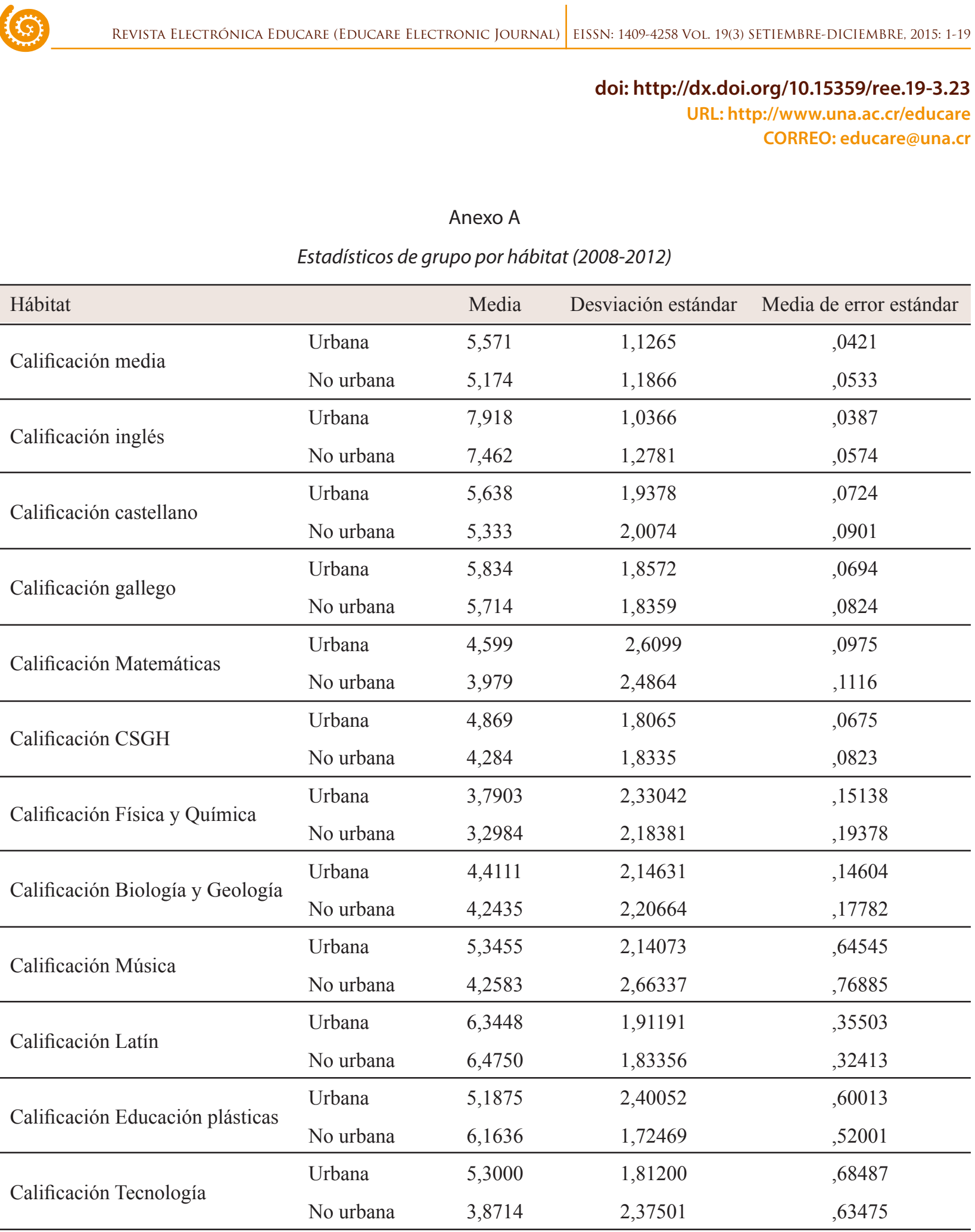


doi: http://dx.doi.org/10.15359/ree.19-3.23

URL: http://www.una.ac.cr/educare

CORREO: educare@una.cr

Anexo B

Prueba de muestras independientes por hábitat para el período (2008-12)

\begin{tabular}{|c|c|c|c|c|c|c|c|c|c|c|}
\hline & & \multicolumn{5}{|c|}{$\begin{array}{l}\text { Prueba de Levene de } \\
\text { calidad de varianzas }\end{array}$} & \multicolumn{4}{|c|}{ prueba t para la igualdad de medias } \\
\hline & & \multirow[b]{2}{*}{$\mathrm{F}$} & \multirow[b]{2}{*}{ Sig. } & \multirow[b]{2}{*}{$t$} & \multirow[b]{2}{*}{$\mathrm{gl}$} & \multirow[b]{2}{*}{$\begin{array}{c}\text { Sig. } \\
\text { (bilateral) }\end{array}$} & \multirow[b]{2}{*}{$\begin{array}{l}\text { Diferencia de } \\
\text { medias }\end{array}$} & \multirow[b]{2}{*}{$\begin{array}{l}\text { Diferencia de } \\
\text { error estándar }\end{array}$} & \multicolumn{2}{|c|}{ 95\% de intervalo de confianza de la diferencia } \\
\hline & & & & & & & & & Inferior & Superior \\
\hline \multirow{2}{*}{$\begin{array}{l}\text { Calificación } \\
\text { media }\end{array}$} & $\begin{array}{l}\text { Se asumen varianzas } \\
\text { iguales }\end{array}$ & ,889 & ,346 & 5,899 & 1210,000 & ,000 & ,397 & ,067 & 265 &, 529 \\
\hline & $\begin{array}{l}\text { No se asumen } \\
\text { varianzas iguales }\end{array}$ & & & 5,844 & 1028,516 & ,000 & ,397 & ,068 & 264 &, 530 \\
\hline \multirow{2}{*}{$\begin{array}{l}\text { Calificación } \\
\text { Inglés }\end{array}$} & $\begin{array}{l}\text { Se asumen varianzas } \\
\text { iguales }\end{array}$ & 20,718 &, 000 & 6,833 & 1210,000 & ,000 & ,456 & ,067 & ,325 & ,587 \\
\hline & $\begin{array}{l}\text { No se asumen } \\
\text { varianzas iguales }\end{array}$ & & & 6,582 & 917,064 & ,000 & ,456 & ,069 & ,320 & ,592 \\
\hline \multirow{2}{*}{$\begin{array}{l}\text { Calificación } \\
\text { Castellano }\end{array}$} & $\begin{array}{l}\text { Se asumen varianzas } \\
\text { iguales }\end{array}$ & 1,240 & ,266 & 2,655 & 1210,000 & ,008 & ,305 & 115 & ,080 & ,530 \\
\hline & $\begin{array}{l}\text { No se asumen } \\
\text { varianzas iguales }\end{array}$ & & & 2,638 & 1040,268 & ,008 & ,305 & 116 & ,078 & ,532 \\
\hline \multirow{2}{*}{$\begin{array}{l}\text { Calificación } \\
\text { Gallego }\end{array}$} & $\begin{array}{l}\text { Se asumen varianzas } \\
\text { iguales }\end{array}$ & 1,333 & 249 & 1,116 & 1210,000 & 265 & 121 & 108 &,- 091 & ,332 \\
\hline & $\begin{array}{l}\text { No se asumen } \\
\text { varianzas iguales }\end{array}$ & & & 1,118 & 1072,436 & 264 & 121 & 108 &,- 091 & ,332 \\
\hline \multirow{2}{*}{$\begin{array}{l}\text { Calificación } \\
\text { Matemáticas }\end{array}$} & $\begin{array}{l}\text { Se asumen varianzas } \\
\text { iguales }\end{array}$ & 4,687 & ,031 & 4,147 & 1210,000 & ,000 & 620 & 150 & ,327 & ,914 \\
\hline & $\begin{array}{l}\text { No se asumen } \\
\text { varianzas iguales }\end{array}$ & & & 4,183 & 1096,689 & ,000 & 620 & 148 & ,329 & 911 \\
\hline \multirow{2}{*}{$\begin{array}{l}\text { Calificación } \\
\text { CSGH }\end{array}$} & $\begin{array}{l}\text { Se asumen varianzas } \\
\text { iguales }\end{array}$ & ,004 & ,948 & 5,509 & 1210,000 & ,000 & ,585 & 106 & ,377 & 793 \\
\hline & $\begin{array}{l}\text { No se asumen } \\
\text { varianzas iguales }\end{array}$ & & & 5,494 & 1054,476 & ,000 & ,585 & 106 & ,376 & ,794 \\
\hline \multirow{2}{*}{$\begin{array}{l}\text { Calificación } \\
\text { Física y Química }\end{array}$} & $\begin{array}{l}\text { Se asumen varianzas } \\
\text { iguales }\end{array}$ & 1,253 & ,264 & 1,961 & 362,000 & ,051 & ,492 & 251 &,- 001 & ,985 \\
\hline & $\begin{array}{l}\text { No se asumen } \\
\text { varianzas iguales }\end{array}$ & & & 2,000 & 272,518 & ,046 & ,492 & 246 & ,008 & ,976 \\
\hline \multirow{2}{*}{$\begin{array}{l}\text { Calificación } \\
\text { Biología y } \\
\text { Geología }\end{array}$} & $\begin{array}{l}\text { Se asumen varianzas } \\
\text { iguales }\end{array}$ & ,014 & 907 & ,732 & 368,000 & ,465 & 168 & 229 &,- 283 & 618 \\
\hline & $\begin{array}{l}\text { No se asumen } \\
\text { varianzas iguales }\end{array}$ & & & ,728 & 324,083 & ,467 & 168 & 230 &,- 285 & ,620 \\
\hline
\end{tabular}

Continúa 


\begin{tabular}{|c|c|c|c|c|c|c|c|c|c|c|}
\hline & & \multicolumn{5}{|c|}{$\begin{array}{l}\text { Prueba de Levene de } \\
\text { calidad de varianzas }\end{array}$} & \multicolumn{4}{|c|}{ prueba t para la igualdad de medias } \\
\hline & & \multirow[b]{2}{*}{$\mathrm{F}$} & \multirow[b]{2}{*}{ Sig. } & \multirow[b]{2}{*}{$\mathrm{t}$} & \multirow[b]{2}{*}{$\mathrm{gl}$} & \multirow{2}{*}{$\begin{array}{c}\text { Sig. } \\
\text { (bilateral) }\end{array}$} & \multirow{2}{*}{$\begin{array}{l}\text { Diferencia de } \\
\text { medias }\end{array}$} & \multirow{2}{*}{$\begin{array}{l}\text { Diferencia de } \\
\text { error estándar }\end{array}$} & \multicolumn{2}{|c|}{ 95\% de intervalo de confianza de la diferencia } \\
\hline & & & & & & & & & Inferior & Superior \\
\hline \multirow{2}{*}{$\begin{array}{l}\text { Calificación } \\
\text { Música }\end{array}$} & $\begin{array}{l}\text { Se asumen varianzas } \\
\text { iguales }\end{array}$ & 1,569 & ,224 & 1,072 & 21,000 & 296 & 1,087 & 1,014 & $-1,021$ & 3,195 \\
\hline & $\begin{array}{l}\text { No se asumen } \\
\text { varianzas iguales }\end{array}$ & & & 1,083 & 20,673 & 291 & 1,087 & 1,004 & $-1,003$ & 3,177 \\
\hline \multirow{2}{*}{$\begin{array}{l}\text { Calificación } \\
\text { Latín }\end{array}$} & $\begin{array}{l}\text { Se asumen varianzas } \\
\text { iguales }\end{array}$ & ,061 &, 805 &,- 271 & 59,000 & ,787 &,- 130 & 480 & $-1,090$ &, 830 \\
\hline & $\begin{array}{l}\text { No se asumen } \\
\text { varianzas iguales }\end{array}$ & & &,- 271 & 57,836 & ,788 &,- 130 & ,481 & $-1,093$ &, 832 \\
\hline \multirow{2}{*}{$\begin{array}{l}\text { Calificación } \\
\text { Educación } \\
\text { plásticas }\end{array}$} & $\begin{array}{l}\text { Se asumen varianzas } \\
\text { iguales }\end{array}$ & ,720 &, 404 & $-1,156$ & 25,000 & 259 &,- 976 &, 844 & $-2,715$ & ,763 \\
\hline & $\begin{array}{l}\text { No se asumen } \\
\text { varianzas iguales }\end{array}$ & & & $-1,229$ & 24,914 & 230 &,- 976 & 794 & $-2,612$ & 660 \\
\hline \multirow{2}{*}{$\begin{array}{l}\text { Calificación } \\
\text { Tecnología }\end{array}$} & $\begin{array}{l}\text { Se asumen varianzas } \\
\text { iguales }\end{array}$ & 1,815 & ,194 & 1,395 & 19,000 & 179 & 1,429 & 1,024 &,- 715 & 3,572 \\
\hline & $\begin{array}{l}\text { No se asumen } \\
\text { varianzas iguales }\end{array}$ & & & 1,530 & 15,468 & 146 & 1,429 & ,934 &,- 557 & 3,414 \\
\hline
\end{tabular}

\section{Cómo citar este artículo en APA:}

Chao-Fernández, R., Ferreiro-Seoane, F. J. y Mato-Vázquez, M. D. (Setiembre-Diciembre, 2015). Análisis de las diferencias de rendimiento del alumnado de centros urbanos y no urbanos a partir de los resultados de los premios extraordinarios de educación secundaria obligatoria. Revista Electrónica Educare, 19(3), 1-19. doi: http://dx.doi.org/10.15359/ree.19-3.23

Nota: Para citar este artículo en otros sistemas puede consultar el hipervínculo "Como citar el artículo" en la barra derecha de nuestro sitio web: http://www.revistas.una.ac.cr/index.php/EDUCARE/index 